\title{
Second-Order Impulsive Differential Equations with Functional Initial Conditions on Unbounded Intervals
}

\author{
Luigi Muglia and Paolamaria Pietramala \\ Dipartimento di Matematica e Informatica, Università della Calabria, 87036 Arcavacata di Rende, Cosenza, Italy \\ Correspondence should be addressed to Luigi Muglia; muglia@mat.unical.it
}

Received 29 December 2012; Accepted 27 February 2013

Academic Editor: Feliz Minhós

Copyright (C) 2013 L. Muglia and P. Pietramala. This is an open access article distributed under the Creative Commons Attribution License, which permits unrestricted use, distribution, and reproduction in any medium, provided the original work is properly cited.

We present some results on the existence of solutions for second-order impulsive differential equations with deviating argument subject to functional initial conditions. Our results are based on Schaefer's fixed point theorem for completely continuous operators.

\section{Introduction}

Differential equations with impulses arise quite often in the study of different problems in particular are used as a model for evolutionary processes subject to a sudden rapid change of their state at certain moments. The theory of impulsive differential equations has become recently a quite active area of research. For an introduction to this theory we refer to the books [1-4], which also contain a variety of interesting examples and applications.

In this paper, we establish new results for the existence of solutions for the second-order impulsive differential equation with deviating argument subject to initial conditions:

$$
\begin{gathered}
x^{\prime \prime}(t)=A x(t)+f(t, x(t), x(\alpha(t))), \\
t \in[0,+\infty) \backslash\left\{t_{1}, \ldots, t_{l}\right\} \\
x\left(t_{k}^{+}\right)-x\left(t_{k}^{-}\right)=I_{k}\left(x\left(t_{k}\right)\right), \quad k=1, \ldots, l \\
x^{\prime}\left(t_{k}^{+}\right)-x^{\prime}\left(t_{k}^{-}\right)=J_{k}\left(x\left(t_{k}\right)\right), \quad k=1, \ldots, l \\
x(t)=\phi(t), \quad t \in[-r, 0], \\
x^{\prime}(0)=\phi^{\prime}(0):=\eta,
\end{gathered}
$$

where $x(t)$ is in the space $\left(\mathbb{R}^{n},|\cdot|\right)(|\cdot|$ not necessarily the Euclidean norm), $\Theta:=\left\{0<t_{1}<\cdots<t_{l}\right\} \subseteq[0,+\infty), A$ is a real $n \times n$ matrix, $x\left(\tau^{-}\right)$, and $x\left(\tau^{+}\right)$are the left and right limits of $x$ in $t=\tau$.
Such type of problem arises from a few of scientific applications as, for example, the problem of impulsive maneuver of a spacecraft (see [5] in finite-dimentional setting and [6] in infinite-dimentional setting). More recently, impulsive second-order differential equations or inclusions on compact intervals subject to nonhomogeneous conditions have been studied by several authors (see for example [7-14] and the references therein). The study of differential problems on unbounded interval has been done, for example, in [14-18].

Differential equations with deviating argument are investigated, for example, in $[6,7,9,12,14,16,17,19,20]$.

Various techniques are utilized in the above papers: Schauder's fixed point Theorem $[6,9,16,17,19,20]$, LeraySchauder's nonlinear alternative $[14,18]$, Contractions Principle $[8,13]$, fixed point index theory [12], and Sadovskii's fixed point Theorem [7]. Moreover, in order to prove the compactness of involved operator, the Ascoli-Arzelà Theorem is often used (see $[6,9,12,14,18]$ ).

The methodology here is to write the problem $(\mathrm{P})$ as a perturbed integral equation, and we look for fixed points of an operator $T$ in a suitable functions space. For this purpose we want to utilize the Schaefer's fixed point theorem for completely continuous operators. In order to prove that $T$ is completely continuous, we make use of a variant of the compactness result in the Banach space $B C\left(Q, \mathbb{R}^{n}\right)$ of continuous bounded functions from a topological space $Q$ into $\mathbb{R}^{n}$ due to De Pascale, Lewicki and G. Marino. We have used similar techniques and tools in $[16,17,20]$ to study first-order, impulsive, or nonimpulsive, differential 
or integrodifferential equations on unbounded intervals. In this paper we extend these ideas to second-order impulsive equations.

Moreover we prove that our method can be easily used also in the case of functional conditions that, to the best of the our knowledge, are not studied for this class of problems. In fact, we discuss in details the Problem (P) in the case of the initial conditions

$$
x(t)=\phi(t) \quad t \in[-r, 0], \quad x^{\prime}(0)=\phi^{\prime}(0)
$$

because it sheds light on the techniques used, but the same approach may be applied to impulsive equations subject to a more general functional initial condition that covers a large number of cases, namely

$$
L x=H(x), \quad x^{\prime}(0)=\eta,
$$

where $L$ is a bounded linear operator and $H$ is a completely continuous operator. This is done in the last section.

\section{Notations and Preliminaries}

Firstly, we recall definitions, notations, and useful facts regarding the cosine families (see [21-23] for the detailed study of cosine and sine families).

Definition 1. A one-parameter family $(C(t))_{t \in \mathbb{R}}$ of bounded linear mappings on $\mathbb{R}^{n}$ into itself is called a strongly continuous cosine family if and only if

(1) $C(t+s)+C(t-s)=2 C(t) C(s)$, for all $t, s \in \mathbb{R}$,

(2) $C(0)=I$, where $I$ is the identity map,

(3) $C(\cdot) x \in C\left(\mathbb{R}, \mathbb{R}^{n}\right)$, for all $x \in \mathbb{R}^{n}$.

Moreover, the sine family $(S(t))_{t \in \mathbb{R}}$ is defined as

$$
S(t):=\int_{0}^{t} C(s) d s .
$$

By definition one obtains that:

(u1) $S(t) x$ is continuous in $t \in \mathbb{R}, S(0)=0$ and $S(-t)=$ $-S(t)$, for all $t \in \mathbb{R}$,

(u2) $C(t)=C(-t)$, for all $t \in \mathbb{R}$,

(u3) $C(t), S(t), S(s), C(s)$ commute, for all $t, s \in \mathbb{R}$,

(u4) $S(t+s)+S(t-s)=2 S(t) C(s)$, for all $t, s \in \mathbb{R}$,

(u5) $S(t+s)=S(t) C(s)+C(t) S(s)$, for all $t, s \in \mathbb{R}$.

Definition 2. The infinitesimal generator of a strongly continuous cosine family is the operator $A: \mathbb{R}^{n} \rightarrow \mathbb{R}^{n}$ defined by

$$
A:=\left.\frac{d^{2}}{d t^{2}} C(t)\right|_{t=0}
$$

and $\operatorname{Dom}(A):=\left\{x \in \mathbb{R}^{n} C(t) x\right.$ is continuously differentiable in $t=0\}$.

One obtains that, for $x \in \mathbb{R}^{n}$ and $t \in \mathbb{R}$,

$$
\frac{d^{2}}{d t^{2}} C(t) x=A C(t) x=C(t) A x
$$

and then

$$
\frac{d}{d t} C(t) x=\int_{0}^{t} A C(s) x d s=A \int_{0}^{t} C(s) x d s=A S(t) x
$$

So, by (u5) one has

(u6) $C(t+s)=C(t) C(s)+A S(t) S(s)$, for all $t, s \in \mathbb{R}$.

Definition 3. A cosine family $(C(t))_{t \in \mathbb{R}}$ (resp., a sine family $(S(t))_{t \in \mathbb{R}}$ ) is uniformly bounded if there exists $M_{C}>0$ (resp., $\left.M_{S}>0\right)$ such that

$$
|C(t)| \leq M_{C} \quad\left(\text { resp. }|S(t)| \leq M_{S}\right) \quad \forall t \in \mathbb{R},
$$

where $|\cdot|$ denotes the norm in the space of the $n \times n$ matrices.

Example 4. In $\mathbb{R}^{n}$, let $(C(t))_{t \in \mathbb{R}}$ be the family of bounded linear operators

$$
C(t)=\left(\begin{array}{cccc}
\cos \left(a_{1} t\right) & 0 & \cdots & 0 \\
0 & \cos \left(a_{2} t\right) & \cdots & 0 \\
\vdots & \vdots & \vdots & \vdots \\
0 & 0 & \cdots & \cos \left(a_{n} t\right)
\end{array}\right) .
$$

Let us observe that $(C(t))_{t \in \mathbb{R}}$ is a uniformly bounded cosine family and its infinitesimal generator is

$$
A=\left.\frac{d^{2}}{d t^{2}} C(t)\right|_{t=0}\left(\begin{array}{cccc}
-a_{1}^{2} & 0 & \ldots & 0 \\
0 & -a_{2}^{2} & \ldots & 0 \\
\vdots & \vdots & \vdots & \vdots \\
0 & 0 & \ldots & -a_{n}^{2}
\end{array}\right)
$$

We will utilize the Schaefer's fixed point theorem for completely continuous operators.

Theorem 5 (see [24]). Let $E$ be a normed linear space. Let $S$ : $E \rightarrow E$ be a completely continuous operator. Let

$$
\zeta(S):=\{x \in E: x=\lambda S x \text { for some } 0<\lambda<1\} \text {. }
$$

Then either $\zeta(S)$ in unbounded or $S$ has a fixed point.

We use the condition for compactness in the Banach space $B C\left(Q, \mathbb{R}^{n}\right)$ of continuous bounded functions $y$ from a topological space $Q$ into $\mathbb{R}^{n}$, endowed with the norm \| $y \|_{\infty}:=\sup \{|y(t)|, t \in Q\}$, due to De Pascale, Lewicki and G. Marino.

Theorem 6 (see [25]). Let $T: B C\left(Q, \mathbb{R}^{n}\right) \rightarrow B C\left(Q, \mathbb{R}^{n}\right)$ be a continuous operator. Suppose that, for any bounded set $F \subset$ $B C\left(Q, \mathbb{R}^{n}\right), T(F)$ is a bounded set and there exist $v$ bounded functions $\varphi_{j}: Q \rightarrow \mathbb{R}^{n}, j=1,2, \ldots, v$, such that, for all $t, s \in Q$ and for all $y \in F$,

$$
|(T y)(t)-(T y)(s)| \leq \sum_{j=1}^{v}\left|\varphi_{j}(t)-\varphi_{j}(s)\right| .
$$

Then $T$ is a compact operator. 


\section{The Integral Problem}

From now on, we assume that the functions $f, \phi, \alpha, I_{k}, J_{k}$ and the matrix $A$ have the following properties.

$\left(h_{0}\right)$ The matrix $A$ is the infinitesimal generator of a strongly continuous cosine family $(C(t))_{t \in \mathbb{R}}$, uniformly bounded by $M_{C}>0$. Suppose moreover that the corresponding sine family $(S(t))_{t \in \mathbb{R}}$ is uniformly bounded by a constant $M_{S}>0$.

$\left(h_{1}\right) f:[0,+\infty) \times \mathbb{R}^{n} \times \mathbb{R}^{n} \rightarrow \mathbb{R}^{n}$ is a continuous function such that there exist a continuous integrable function $p:[0,+\infty) \rightarrow[0,+\infty)$ and a continuous nondecreasing function $\Psi:[0,+\infty) \rightarrow[1,+\infty)$ for which

$$
|f(t, x, y)| \leq p(t) \Psi(|x|+|y|), \quad t \geq 0, x, y \in \mathbb{R}^{n},
$$

$$
\int_{0}^{\infty} \frac{d s}{\Psi(s)}=+\infty
$$

$\left(h_{2}\right) \alpha:[0,+\infty) \rightarrow[-r,+\infty)$ is a continuous increasing function such that $\alpha(t) \leq t$, for all $t \in[0,+\infty)$.

The function $\phi$ belongs to $C^{1}\left([-r, 0], \mathbb{R}^{n}\right)$.

$\left(h_{3}\right) I_{k}: \mathbb{R}^{n} \rightarrow \mathbb{R}^{n}$ and $J_{k}: \mathbb{R}^{n} \rightarrow \mathbb{R}^{n}, k=1, \ldots, l$ are continuous functions such that there are $D_{I}, D_{J}>0$ for which

$$
\left|I_{k}(v)\right| \leq D_{I}, \quad\left|J_{k}(v)\right| \leq D_{J}, \quad \forall v \in \mathbb{R}^{n} .
$$

We will work in the Banach space

$$
\begin{aligned}
& B P C_{\Theta}[-r,+\infty) \\
& :=\left\{y:[-r,+\infty) \longrightarrow \mathbb{R}^{n} \mid y\right. \text { is } \\
& \quad \text { and continuous in } t \notin \Theta, \\
& \text { there exist } y\left(t_{k}^{-}\right)=y\left(t_{k}\right) \\
& \left.\quad \text { and } y\left(t_{k}^{+}\right)<\infty, t_{k} \in \Theta\right\},
\end{aligned}
$$$$
:=\left\{y:[-r,+\infty) \longrightarrow \mathbb{R}^{n} \mid y\right. \text { is bounded }
$$

endowed with the supremum norm $\|y\|_{\infty}=\sup \{|y(t)|: t \epsilon$ $[-r,+\infty)\}$.

We define for $x \in B P C_{\Theta}[-r,+\infty)$ :

$$
\begin{aligned}
w_{x}(t):= \begin{cases}\int_{0}^{t} S(t-s) f(s, x(s), x(\alpha(s))) d s, & t \geq 0, \\
0, & -r \leq t \leq 0\end{cases} \\
(I x)(t):= \begin{cases}\sum_{0<t_{k}<t} C\left(t-t_{k}\right) I_{k}\left(x\left(t_{k}\right)\right), & t>t_{1}, \\
0, & -r \leq t \leq t_{1}\end{cases} \\
(J x)(t):= \begin{cases}\sum_{0<t_{k}<t} S\left(t-t_{k}\right) J_{k}\left(x\left(t_{k}\right)\right), & t>t_{1}, \\
0, & -r \leq t \leq t_{1} .\end{cases}
\end{aligned}
$$

For any $x \in B P C_{\Theta}[-r,+\infty)$ let $T x$ be the function defined on $[-r,+\infty)$ by

$$
(T x)(t):=\left\{\begin{array}{l}
C(t) \phi(0)+S(t) \eta+w_{x}(t) \\
+(I x)(t)+(J x)(t), \quad t \geq 0 \\
\phi(t), \quad-r \leq t \leq 0 .
\end{array}\right.
$$

In the next propositions we show the properties of the operator $T$ useful for us.

Proposition 7. The operator $T$ maps $B P C_{\Theta}[-r,+\infty)$ into $B P C_{\Theta}[-r,+\infty)$ and $(T x)\left(t_{k}^{+}\right)-(T x)\left(t_{k}^{-}\right)=I_{k}\left(x\left(t_{k}\right)\right)$. Moreover Tx has derivative in $t \in[-r,+\infty) \backslash \Theta,(T x)^{\prime}\left(t_{k}^{-}\right)=(T x)^{\prime}\left(t_{k}\right)$, $(T x)^{\prime}\left(t_{k}^{+}\right)<\infty$ and $(T x)^{\prime}\left(t_{k}^{+}\right)-(T x)^{\prime}\left(t_{k}^{-}\right)=J_{k}\left(x\left(t_{k}\right)\right)$.

Proof. The continuity of $f, S$, and $C$ guarantees that $T x$ is continuous in $t \in[-r,+\infty) \backslash \Theta$, and there exists $(T x)^{\prime}(t)$ for $t \in[-r,+\infty) \backslash \Theta$. Moreover, since $p$ is integrable, for $t \in[-r,+\infty) \backslash \Theta$, one has

$$
\begin{aligned}
|(T x)(t)| \leq & M_{C}|\phi(0)|+M_{S}|\eta|+M_{S} \Psi\left(2\|x\|_{\infty}\right) \\
& \times \int_{0}^{\infty} p(s) d s+l M_{C} D_{I}+l M_{S} D_{J},
\end{aligned}
$$

so $\|T x\|_{\infty}<\infty$. We need to show that, for $k=1, \ldots, l$, there exist $(T x)\left(t_{k}^{+}\right)$and $(T x)\left(t_{k}^{-}\right)=(T x)\left(t_{k}\right)$. Note that

(i) $\lim _{t \rightarrow t_{k}} w_{x}(t)=w_{x}\left(t_{k}\right)$. Indeed, using (u5), for $t \rightarrow$ $t_{k}$

$$
\begin{aligned}
& \left|w_{x}(t)-w_{x}\left(t_{k}\right)\right| \\
& \leq \int_{0}^{t}\left|S(t-s)-S\left(t_{k}-s\right)\right| \\
& \quad \cdot|f(s, x(s), x(\alpha(s)))| d s \\
& \quad+\int_{t}^{t_{k}}\left|S\left(t_{k}-s\right)\right| \cdot|f(s, x(s), x(\alpha(s)))| d s \\
& \left(\operatorname{by}\left(h_{1}\right)\right) \\
& \leq \int_{0}^{t}\left|S(t-s)-S\left(t_{k}-s\right)\right| \Psi\left(2\|x\|_{\infty}\right) p(s) d s \\
& \quad+M_{S} \Psi\left(2\|x\|_{\infty}\right) \int_{t}^{t_{k}} p(s) d s \\
& \leq\left(\left|S(t)-S\left(t_{k}\right)\right|+\left|C(t)-C\left(t_{k}\right)\right|\right) \\
& \quad \times \max \left\{M_{S}, M_{C}\right\} \Psi\left(2\|x\|_{\infty}\right) \int_{0}^{t} p(s) d s \\
& \quad+M_{S} \Psi\left(2\|x\|_{\infty}\right) \int_{t}^{t_{k}} p(s) d s \longrightarrow 0 .
\end{aligned}
$$


(ii) It results that $(I x)\left(t_{k}^{-}\right)=(I x)\left(t_{k}\right)=\sum_{j=1}^{k-1} C\left(t_{k}-t_{j}\right)$ $I_{j}\left(x\left(t_{j}\right)\right)$. Indeed let $t_{k-1}<t<t_{k}$. Then

$$
\begin{aligned}
\mid(I x)(t) & -(I x)\left(t_{k}\right) \mid \\
= & \mid \sum_{0<t_{j}<t} C\left(t-t_{j}\right) I_{j}\left(x\left(t_{j}\right)\right) \\
& \quad-\sum_{0<t_{j}<t_{k}} C\left(t_{k}-t_{j}\right) I_{j}\left(x\left(t_{j}\right)\right) \mid,
\end{aligned}
$$

(by $\left.\left(h_{3}\right)\right)$

$$
\leq D_{I} \sum_{j=1}^{k-1}\left|C\left(t-t_{j}\right)-C\left(t_{k}-t_{j}\right)\right| \longrightarrow 0 .
$$

Analogously one can see that

$$
(I x)\left(t_{k}^{+}\right)=\sum_{j=1}^{k} C\left(t_{k}-t_{j}\right) I_{j}\left(x\left(t_{j}\right)\right)
$$

in such a way that $(I x)\left(t_{k}^{+}\right)-(I x)\left(t_{k}^{-}\right)=I_{k}\left(x\left(t_{k}\right)\right)$.

Since, by (u1), $S\left(t_{k}-t_{k}\right)=0$, a similar proof permits to verify that

$$
\begin{aligned}
(J x)\left(t_{k}^{+}\right)-(J x)\left(t_{k}^{-}\right)= & \sum_{j=1}^{k} S\left(t_{k}-t_{j}\right) J_{j}\left(x\left(t_{j}\right)\right) \\
& +\sum_{j=1}^{k-1} S\left(t_{k}-t_{j}\right) J_{j}\left(x\left(t_{j}\right)\right)=0 .
\end{aligned}
$$

By the above steps, it follows that $(T x)\left(t_{k}^{+}\right)-(T x)\left(t_{k}^{-}\right)=$ $I_{k}\left(x\left(t_{k}\right)\right)$.

Since there exists $(T x)^{\prime}(t)$ if $t \in[0,+\infty) \backslash \Theta$, to conclude our proof we show that there exists $(T x)^{\prime}\left(t_{k}^{-}\right)=(T x)^{\prime}\left(t_{k}\right)$, $(T x)^{\prime}\left(t_{k}^{+}\right)$is finite, and $(T x)^{\prime}\left(t_{k}^{+}\right)-(T x)^{\prime}\left(t_{k}^{-}\right)=J_{k}\left(x\left(t_{k}\right)\right)$. We also observe that, for $t \geq 0$ and $t \notin \Theta$, by (u6),

$$
\begin{aligned}
(T x)^{\prime}(t)= & A S(t) \phi(0)+C(t) \eta \\
& +\int_{0}^{t} C(t-s) f(s, x(s), x(\alpha(s))) d s \\
& +\sum_{0<t_{k}<t} A S\left(t-t_{k}\right) I_{k}\left(x\left(t_{k}\right)\right) \\
& +\sum_{0<t_{k}<t} C\left(t-t_{k}\right) J_{k}\left(x\left(t_{k}\right)\right) .
\end{aligned}
$$

Thus, following the same idea, when $t \rightarrow t_{k}$, we have

$$
\begin{aligned}
& \quad \mid \int_{0}^{t} C(t-s) f(s, x(s), x(\alpha(s))) d s \\
& -\int_{0}^{t_{k}} C\left(t_{k}-s\right) f(s, x(s), x(\alpha(s))) d s \mid \\
& \quad \leq \int_{0}^{t}\left|C(t-s)-C\left(t_{k}-s\right)\right| p(s) \Psi\left(2\|x\|_{\infty}\right) d s \\
& \quad+M_{C} \Psi\left(2\|x\|_{\infty}\right) \int_{t}^{t_{k}} p(s) d s \longrightarrow 0 .
\end{aligned}
$$

Moreover, for $t \rightarrow t_{k}^{-}$

$$
\begin{aligned}
& \mid \sum_{0<t_{j}<t} A S\left(t-t_{j}\right) I_{j}\left(x\left(t_{j}\right)\right) \\
& \quad-\sum_{j=1}^{k-1} A S\left(t_{k}-t_{j}\right) I_{j}\left(x\left(t_{j}\right)\right) \mid \\
& \quad \leq D_{I}|A| \sum_{j=1}^{k-1}\left|S\left(t-t_{j}\right)-S\left(t_{k}-t_{j}\right)\right| \longrightarrow 0, \\
& \lim _{t \rightarrow t_{k}^{+}}\left(\sum_{0<t_{k}<t} A S\left(t-t_{k}\right) I_{k}\left(x\left(t_{k}\right)\right)\right) \\
& =\sum_{j=1}^{k-1} A S\left(t-t_{k}\right) I_{j}\left(x\left(t_{j}\right)\right) .
\end{aligned}
$$

Finally, for $t \rightarrow t_{k}^{-}$

$$
\begin{aligned}
& \mid \sum_{0<t_{k}<t} C\left(t-t_{k}\right) J_{k}\left(x\left(t_{k}\right)\right) \\
& -\sum_{j=1}^{k-1} C\left(t_{k}-t_{j}\right) J_{j}\left(x\left(t_{j}\right)\right) \mid \\
& \quad \leq D_{J} \sum_{j=1}^{k-1}\left|S\left(t-t_{j}\right)-S\left(t_{k}-t_{j}\right)\right| \longrightarrow 0,
\end{aligned}
$$

while

$$
\begin{gathered}
\lim _{t \rightarrow t_{k}^{+}}\left(\sum_{0<t_{k}<t} C\left(t-t_{k}\right) J_{k}\left(x\left(t_{k}\right)\right)\right) \\
=\sum_{j=1}^{k} C\left(t-t_{k}\right) J_{j}\left(x\left(t_{j}\right)\right),
\end{gathered}
$$

and these permit to obtain that there exist $(T x)^{\prime}\left(t_{k}^{+}\right)$ and $(T x)^{\prime}\left(t_{k}^{-}\right)$and that one has $(T x)^{\prime}\left(t_{k}^{+}\right)-(T x)^{\prime}\left(t_{k}^{-}\right)=$ $J_{k}\left(x\left(t_{k}\right)\right)$.

Proposition 8. The fixed points of $T$ are solutions of the problem $(P)$. 
Proof. In fact, $\left.T x\right|_{[-r, 0]}=\phi$ and $(T x)^{\prime}(0)=\eta$. Moreover, by the hypotheses on $f$ and on the sine and cosine families, one obtains that $(T x)^{\prime}$ is derivable in $t \in[-r,+\infty) \backslash \Theta$, and it results in the following:

$$
\begin{aligned}
(T x)^{\prime \prime}(t)= & A C(t) \phi(0)+A S(t) \eta \\
& +\int_{0}^{t} A S(t-s) f(s, x(s), x(\alpha(s))) d s \\
& +f(t, x(t), x(\alpha(t))) \\
& +\sum_{0<t_{k}<t} A C\left(t-t_{k}\right) I_{k}\left(x\left(t_{k}\right)\right) \\
& +\sum_{0<t_{k}<t} A S\left(t-t_{k}\right) J_{k}\left(x\left(t_{k}\right)\right) .
\end{aligned}
$$

So one can conclude that

$$
(T x)^{\prime \prime}(t)=A T x(t)+f(t, x(t), x(\alpha(t))),
$$

that is, $T x$ satisfies the differential equation in problem $(\mathrm{P})$. This combined with the results in Proposition 7 yields the thesis.

In order to prove that $T$ is a completely continuous operator, we make use of the following compactness criterion in $B P C_{\Theta}[-r,+\infty)$.

Proposition 9 (see [16]). Let $T: B P C_{\Theta}[-r,+\infty) \rightarrow$ $B P C_{\Theta}[-r,+\infty)$ be a continuous operator. Suppose that, for any bounded set $F \subset B P C_{\Theta}[-r,+\infty), T(F)$ is a bounded set, and there exist $\nu$ bounded functions $\varphi_{j}:[-r,+\infty) \rightarrow \mathbb{R}^{n}$, $j=1,2, \ldots, v$, such that, for all $t, s \in[-r,+\infty)$ and, for all $y \in F$

$$
|(T y)(t)-(T y)(s)| \leq \sum_{j=1}^{v}\left|\varphi_{j}(t)-\varphi_{j}(s)\right| .
$$

\section{Then $T$ is a compact operator.}

Proof. The Banach space $B P C_{\Theta}[-r,+\infty)$ is isometric to the Banach space:

$$
\begin{gathered}
\widetilde{\Omega}=\left\{y \in B C\left(Q, \mathbb{R}^{n}\right): \text { there exist } y\left(t_{k}^{+}\right)\right. \\
\text {and } \left.y\left(t_{k}^{-}\right), \text {for } k=1, \ldots, l\right\},
\end{gathered}
$$

where $Q=[-r,+\infty) \backslash \Theta$. Of course, $\widetilde{\Omega}$ is closed in $B C\left(Q, \mathbb{R}^{n}\right)$. So the thesis follows from Theorem 6 .

\section{Proposition 10. $T$ is a completely continuous operator.}

Proof. First we prove that $T$ is a continuous operator. Let $\left(y_{m}\right)_{m \in \mathbb{N}}$ a sequence in $B P C_{\Theta}[-r,+\infty)$ such that $y_{m} \rightarrow y$ in $B P C_{\Theta}[-r,+\infty)$. We prove that $T y_{m} \rightarrow T y$. By the continuity of $f$ it results that, for fixed $s \in[0,+\infty)$,

$$
\begin{aligned}
\sigma_{m}(s):= & f\left(s, y_{m}(s), y_{m}(\alpha(s))\right) \\
& -f(s, y(s), y(\alpha(s))) \longrightarrow 0 .
\end{aligned}
$$

Moreover, by the monotonicity of $\Psi$, we have

$$
\begin{aligned}
\left|\sigma_{m}(s)\right| \leq & \left|f\left(s, y_{m}(s), y_{m}(\alpha(s))\right)\right| \\
& +|f(s, y(s), y(\alpha(s)))| \\
\leq & p(s)\left[\Psi\left(2\left\|y_{m}\right\|_{\infty}\right)+\Psi\left(2\|y\|_{\infty}\right)\right] \\
\leq & 2 p(s) \Psi(2 \Lambda),
\end{aligned}
$$

where $\Lambda=\max \left\{\sup _{m \in \mathbb{N}}\left\|y_{m}\right\|_{\infty},\|y\|_{\infty}\right.$. Therefore $\left|\sigma_{m}(s)\right|$ is dominated by an integrable function that does not depend on $m \in \mathbb{N}$. Then, from the uniform boundedness of the sine family and by the dominated convergence theorem, we have

$$
\begin{aligned}
\left\|w_{y_{m}}-w_{y}\right\|_{\infty} & =\sup _{0 \leq t<+\infty}\left|w_{y_{m}}(t)-w_{y}(t)\right| \\
& =\sup _{0 \leq t<+\infty}\left|\int_{0}^{t} S(t-s) \sigma_{m}(s) d s\right| \\
& \leq M_{S} \int_{0}^{\infty}\left|\sigma_{m}(s)\right| d s \longrightarrow 0 .
\end{aligned}
$$

The uniform boundedness of the cosine family and the continuity of $I_{k}$ and $J_{k}$ imply that, as $m \rightarrow \infty$,

$$
\begin{gathered}
\left\|I y_{m}-I y\right\|_{\infty} \\
\leq \sup _{t \in\left[t_{1},+\infty\right)}\left(\sum_{0<t_{k}<t}\left|C\left(t-t_{k}\right)\right|\left|I_{k}\left(y_{m}\left(t_{k}\right)\right)-I_{k}\left(y\left(t_{k}\right)\right)\right|\right) \\
\leq M_{C} \sum_{k=1}^{l}\left|I_{k}\left(y_{m}\left(t_{k}\right)\right)-I_{k}\left(y\left(t_{k}\right)\right)\right| \longrightarrow 0, \\
\left\|J y_{m}-J y\right\|_{\infty} \longrightarrow 0 .
\end{gathered}
$$

Thus

$$
\begin{aligned}
\left\|T y_{m}-T y\right\|_{\infty} \leq & \left\|w_{y_{m}}-w_{y}\right\|_{\infty} \\
& +\left\|J y_{m}-J y\right\|_{\infty}+\left\|I y_{m}-I y\right\|_{\infty} \longrightarrow 0 .
\end{aligned}
$$

Now, let

$$
B_{\rho}:=\left\{u \in B P C_{\Theta}[-r,+\infty):\|u\|_{\infty} \leq \rho\right\} .
$$

Thanks to Proposition 9, it is enough to show that $T\left(B_{\rho}\right)$ is a bounded set and that it is possible to control the oscillations of each function in $T\left(B_{\rho}\right)$ by means of a finite number of bounded functions. The boundedness of $T\left(B_{\rho}\right)$ follows by the inequalities:

$$
\begin{gathered}
\left\|w_{x}\right\|_{\infty} \leq M_{S} \Psi(2 \rho) \int_{0}^{\infty} p(s) d s, \\
\|I x\|_{\infty} \leq M_{C} \sum_{k=1}^{l}\left|I_{k}\left(x\left(t_{k}\right)\right)\right| \leq l M_{C} D_{I}, \\
\|J x\|_{\infty} \leq M_{S} \sum_{k=1}^{l}\left|J_{k}\left(x\left(t_{k}\right)\right)\right| \leq l M_{S} D_{J} .
\end{gathered}
$$


To control the oscillations of $T\left(B_{\rho}\right)$ we should need to distinguish three cases: $0 \leq \tau_{1}<\tau_{2}, \tau_{1}<0<\tau_{2}$, and $\tau_{1}<\tau_{2} \leq 0$.

Here we study the case $0 \leq \tau_{1}<\tau_{2}$ only because the proofs of the other cases are similar.

Let $0 \leq \tau_{1}<\tau_{2}$ and $x \in B_{\rho}$. Then

$$
\begin{aligned}
\mid(T x) & \left(\tau_{1}\right)-(T x)\left(\tau_{2}\right) \mid \\
\leq & \left|C\left(\tau_{1}\right)-C\left(\tau_{2}\right)\right||\phi(0)|+\left|S\left(\tau_{1}\right)-S\left(\tau_{2}\right)\right||\eta| \\
& +\left|\int_{0}^{\tau_{1}}\left[S\left(\tau_{1}-s\right)-S\left(\tau_{2}-s\right)\right] f(s, x(s), x(\alpha(s)))\right| d s \\
& +\left|\int_{\tau_{1}}^{\tau_{2}} S\left(\tau_{2}-s\right) f(s, x(s), x(\alpha(s))) d s\right| \\
& +\left|(I x)\left(\tau_{1}\right)-(I x)\left(\tau_{2}\right)\right|+\left|(J x)\left(\tau_{1}\right)-(J x)\left(\tau_{2}\right)\right| .
\end{aligned}
$$

Now, by property (u5), one obtains that

$$
\begin{aligned}
& S\left(\tau_{2}-s\right)-S\left(\tau_{1}-s\right) \\
& \quad=\left[S\left(\tau_{2}\right)-S\left(\tau_{1}\right)\right] C(s)-\left[C\left(\tau_{2}\right)-C\left(\tau_{1}\right)\right] S(s),
\end{aligned}
$$

so we have

$$
\begin{aligned}
& \left|\int_{0}^{\tau_{1}}\left[S\left(\tau_{1}-s\right)-S\left(\tau_{2}-s\right)\right] f(s, x(s), x(\alpha(s))) d s\right| \\
& \leq\left|S\left(\tau_{2}\right)-S\left(\tau_{1}\right)\right| \\
& \quad \times \int_{0}^{\tau_{1}}|C(s)||f(s, x(s), x(\alpha(s)))| d s \\
& \quad+\left|C\left(\tau_{2}\right)-C\left(\tau_{1}\right)\right| \\
& \quad \times \int_{0}^{\tau_{1}}|S(s)||f(s, x(s), x(\alpha(s)))| d s \\
& \leq\left|S\left(\tau_{2}\right)-S\left(\tau_{1}\right)\right| M_{C} \Psi(2 \rho) \int_{0}^{\infty} p(s) d s \\
& \quad+\left|C\left(\tau_{2}\right)-C\left(\tau_{1}\right)\right| M_{S} \Psi(2 \rho) \int_{0}^{\infty} p(s) d s .
\end{aligned}
$$

Using $\left(h_{3}\right)$ we obtain also that

$$
\begin{aligned}
& \left|\int_{\tau_{1}}^{\tau_{2}} S\left(\tau_{2}-s\right) f(s, x(s), x(\alpha(s))) d s\right| \\
& \quad \leq M_{S} \Psi(2 \rho)\left[\int_{0}^{\tau_{2}} p(s) d s-\int_{0}^{\tau_{1}} p(s) d s\right] .
\end{aligned}
$$

To control the oscillations of the operators $I$ and $J$, first we note that if $\tau_{1}<t_{1}$, and $\tau_{2}<t_{1}$ we have nothing to prove because $(I x)\left(\tau_{1}\right)=(I x)\left(\tau_{2}\right)=(J x)\left(\tau_{1}\right)=(J x)\left(\tau_{2}\right)=0$. The boundedness of $I$ and $J$ solves also the case $\tau_{1}<t_{1}<\tau_{2}$ since we have

$$
\begin{aligned}
& \left|(I x)\left(\tau_{2}\right)-(I x)\left(\tau_{1}\right)\right|=\left|(I x)\left(\tau_{2}\right)\right| \leq D_{I} \sum_{0<t_{k}<\tau_{2}} M_{C}, \\
& \left|(J x)\left(\tau_{2}\right)-(J x)\left(\tau_{1}\right)\right|=\left|(J x)\left(\tau_{2}\right)\right| \leq D_{J} \sum_{0<t_{k}<\tau_{2}} M_{S} .
\end{aligned}
$$

Thus it remains to prove only the case $t_{1}<\tau_{1}<\tau_{2}$. We have that

$$
\begin{aligned}
\mid(I x) & \left(\tau_{1}\right)-(I x)\left(\tau_{2}\right) \mid \\
= & \mid \sum_{0<t_{k}<\tau_{1}} C\left(\tau_{1}-t_{k}\right) I_{k}\left(x\left(t_{k}\right)\right) \\
& -\sum_{0<t_{k}<\tau_{2}} C\left(\tau_{2}-t_{k}\right) I_{k}\left(x\left(t_{k}\right)\right) \mid \\
\leq & \left|\sum_{0<t_{k}<\tau_{1}}\left[C\left(\tau_{1}-t_{k}\right)-C\left(\tau_{2}-t_{k}\right)\right] I_{k}\left(x\left(t_{k}\right)\right)\right| \\
& +\sum_{\tau_{1} \leq t_{k}<\tau_{2}}\left|C\left(\tau_{2}-t_{k}\right)\right|\left|I_{k}\left(x\left(t_{k}\right)\right)\right| \\
\leq & D_{I} \sum_{k=1}^{l}\left|C\left(\tau_{1}-t_{k}\right)-C\left(\tau_{2}-t_{k}\right)\right| \\
& +D_{I}\left[\sum_{0<t_{k}<\tau_{2}} M_{C}-\sum_{0<t_{k}<\tau_{1}} M_{C}\right] .
\end{aligned}
$$

In a similar way we can show that

$$
\begin{aligned}
& \left|(J x)\left(\tau_{1}\right)-(J x)\left(\tau_{2}\right)\right| \\
& \leq D_{J} \sum_{k=1}^{l}\left|S\left(\tau_{1}-t_{k}\right)-S\left(\tau_{2}-t_{k}\right)\right| \\
& \quad+D_{J}\left[\sum_{0<t_{k}<\tau_{2}} M_{S}-\sum_{0<t_{k}<\tau_{1}} M_{S}\right] .
\end{aligned}
$$

So, defining

$$
\begin{gathered}
\psi_{1}(t)=C(t) \phi(0), \quad \psi_{2}(t)=S(t) \eta, \\
\psi_{3}(t)=M_{C} K S(t), \quad \psi_{4}(t)=M_{S} K C(t), \\
\psi_{5}(t)=M_{S} \Psi(2 \rho) \int_{0}^{t} p(s) d s, \\
\psi_{6}(t)=D_{I} C\left(t-t_{1}\right), \\
\psi_{7}(t)=D_{I} C\left(t-t_{2}\right), \ldots, \psi_{l+5}(t)=D_{I} C\left(t-t_{l}\right), \\
\psi_{l+6}(t)=D_{I} M_{C} \sum_{0<t_{k}<t} 1, \\
\psi_{l+7}(t)=D_{J} S\left(t-t_{1}\right), \\
\psi_{l+8}(t)=D_{J} S\left(t-t_{2}\right), \ldots, \psi_{2 l+6}(t)=D_{J} S\left(t-t_{l}\right), \\
\psi_{2 l+7}(t)=D_{J} M_{S} \sum_{0<t_{k}<t} 1,
\end{gathered}
$$

where $K=\Psi(2 \rho) \int_{0}^{\infty} p(s) d s$, and we obtain that for all $x \in B_{\rho}$

$$
\left|(T x)\left(\tau_{1}\right)-(T x)\left(\tau_{2}\right)\right| \leq \sum_{k=1}^{2 l+7}\left|\psi_{k}\left(\tau_{1}\right)-\psi_{k}\left(\tau_{2}\right)\right| .
$$




\section{Main Result}

Theorem 11. Assume that the hypotheses $\left(h_{0}\right)-\left(h_{3}\right)$ hold. Then the problem $(P)$ has at least one solution.

Proof. Our problem (P) can be reduced, by Propositions 7 and 8 , to find a fixed point for the operator $T$. Proposition 10 assures that $T$ is a completely continuous operator.

To apply the Schaefer's fixed point theorem, it remains to prove that the set

$$
\zeta(T):=\left\{x \in \mathbb{R}^{n}: x=\lambda T x \text {, for some } 0<\lambda<1\right\}
$$

is bounded. The proof is based on an idea in [19].

Let $x=\lambda(T x)$ with $\lambda \in(0,1)$. First of all, since $\left\{\left.T x\right|_{[-r, 0]}\right.$ : $\left.x \in B P C_{\Theta}[-r,+\infty)\right\}=\{\phi\}$, for $t \in[-r, 0]$ we have

$$
|x(t)|=\lambda|(T x)(t)|<|(T x)(t)|=|\phi(t)| \leq\|\phi\|_{\infty} .
$$

We consider now that $t \geq 0$. Thus we have

$$
\begin{aligned}
|x(t)|= & \lambda|(T x)(t)|<M_{C}\|\phi\|_{\infty}+M_{S}|\eta| \\
& +M_{S} \int_{0}^{t} p(s) \Psi(|x(s)|+|x(\alpha(s))|) d s \\
& +l M_{C} D_{I}+l M_{S} D_{J} .
\end{aligned}
$$

Consider the function $\mu_{x}:[0,+\infty) \rightarrow[0,+\infty)$ defined by

$$
\mu_{x}(t):=\sup \{|x(\xi)|: 0 \leq \xi \leq t\}
$$

Observe that $\mu_{x}$ is not necessarily continuous in $\Theta$ but $\mu_{x}\left(t_{k}^{+}\right)$ and $\mu_{x}\left(t_{k}^{-}\right)=\mu_{x}\left(t_{k}\right)$ exist, for all $t_{k} \in \Theta$.

For $\xi \in[0, t]$, we have

$$
\begin{aligned}
|x(\xi)| \leq & M_{C}\|\phi\|_{\infty}+M_{S}|\eta| \\
& +M_{S} \int_{0}^{t} p(s) \Psi(|x(s)|+|x(\alpha(s))|) d s \\
& +l M_{S} D_{I}+l M_{C} D_{J} .
\end{aligned}
$$

Moreover, being $\alpha$ increasing and $\alpha(t) \leq t$ for $t \geq 0$, one has

$$
\begin{aligned}
\sup _{0 \leq s \leq t}|x(\alpha(s))| & =\sup _{\alpha(0) \leq s \leq \alpha(t)}|x(s)| \\
& \leq \sup _{0 \leq s \leq t}|x(s)|=\mu_{x}(t)
\end{aligned}
$$

so, taking the supremum over $[0, t]$ in the inequality (52), we obtain that

$$
\begin{aligned}
\mu_{x}(t) \leq & M_{C}\|\phi\|_{\infty}+M_{S}|\eta| \\
& +M_{S} \int_{0}^{t} p(s) \Psi\left(2 \mu_{x}(t)\right) d s \\
& +l M_{S} D_{I}+l M_{C} D_{J} .
\end{aligned}
$$

Denoting by $v_{x}(t)$ the right-hand side of the last inequality, we have that the function $v_{x}$ is continuous:

$$
c:=v_{x}(0)=\left(1+M_{C}\right)\|\phi\|_{\infty}+M_{S}|\eta|+l M_{S} D_{I}+l M_{C} D_{J}
$$

and $\mu_{x}(t) \leq v_{x}(t)$ for $t \geq 0$.

Moreover, since $\Psi$ is nondecreasing, for $t \notin \Theta$,

$$
v_{x}^{\prime}(t) \leq M_{S} p(t) \Psi\left(2 v_{x}(t)\right)
$$

This implies that

$$
\frac{v_{x}^{\prime}(t)}{\Psi\left(2 v_{x}(t)\right)} \leq M_{S} p(t), \quad t \notin \Theta
$$

and so, for any $b>0$,

$$
\int_{0}^{b} \frac{v_{x}^{\prime}(t)}{\Psi\left(2 v_{x}(t)\right)} d t \leq M_{S} \int_{0}^{b} p(t):=\Gamma_{b}<\infty .
$$

Since $v_{x}^{\prime}$ is a continuous function for all $t \notin \Theta$, we have

$$
\int_{2 c}^{2 v_{x}(b)} \frac{d s}{2 \Psi(s)} \leq \Gamma_{b} .
$$

This, together with condition (13), permits us to conclude that $v_{x}$ is bounded by a constant $\Delta$ depending on the functions $\Psi$ and $p$ only. $\Delta\}$.

Summarizing, $x \in \zeta(T)$ implies that $\|x\|_{\infty} \leq \max \left\{\|\phi\|_{\infty}\right.$,

\section{Functional Initial Conditions}

Let us consider the problem

$$
\begin{gathered}
x^{\prime \prime}(t)=A x(t)+f(t, x(t), x(\alpha(t))), \\
t \in[0,+\infty) \backslash\left\{t_{1}, \ldots, t_{l}\right\} \\
x\left(t_{k}^{+}\right)-x\left(t_{k}^{-}\right)=I_{k}\left(x\left(t_{k}\right)\right), \quad k=1, \ldots, l \\
x^{\prime}\left(t_{k}^{+}\right)-x^{\prime}\left(t_{k}^{-}\right)=J_{k}\left(x\left(t_{k}\right)\right), \quad k=1, \ldots, l \\
L x=H(x), \quad x^{\prime}(0)=\eta .
\end{gathered}
$$

The modularity of the operator $T$ used in the proof of our Theorem 11 permits to prove a result on the existence of solutions for Problem $\left(\mathrm{P}_{\mathrm{F}}\right)$ modifying few parts of the above proof.

For $u \in B P C_{\Theta}[-r,+\infty)$, we define the function $\widetilde{C} u$ : $[-r,+\infty) \rightarrow \mathbb{R}^{n}$ as

$$
(\widetilde{C} u)(t):= \begin{cases}u(t), & -r \leq t \leq 0 \\ C(t) u(0), & t \geq 0\end{cases}
$$

We suppose that

$\left(h_{4}\right) L: B P C_{\Theta}[-r,+\infty) \rightarrow C\left([-r, 0], \mathbb{R}^{n}\right)$ is a bounded linear operator for which if $u, v \in B P C_{\Theta}[-r,+\infty)$ are such that $\left.u\right|_{[-r, 0]}=\left.v\right|_{[-r, 0]}$, then $L u=L v$. 
$H: B P C_{\Theta}[-r,+\infty) \rightarrow C\left([-r, 0] ; \mathbb{R}^{n}\right)$ is a completely continuous operator such that $\|H(u)\|_{\infty} \leq M_{1}$ for a certain constant $M_{1}$.

$\left(h_{5}\right)$ For $u \in B P C_{\Theta}[-r,+\infty), H(u)=(L \widetilde{C} H)(u)$.

Theorem 12. Assume that the hypotheses $\left(h_{0}\right)-\left(h_{5}\right)$ hold. Then the problem $\left(P_{F}\right)$ has at least one solution.

Proof. Let us consider the operator $W$ defined on $B P C_{\Theta}[-r,+\infty)$ as

$$
\begin{aligned}
(W x)(t):= & (\widetilde{C} H(x))(t)+S(t) \eta \\
& +w_{x}(t)+(I x)(t)+(J x)(t) .
\end{aligned}
$$

Following the proof of Theorem 11, it is necessary to study only the part of the operator arising from the functional initial condition. First, for $t \geq 0$, one has

$$
\begin{aligned}
|(\widetilde{C} H(x))(t)| & \leq M_{C}|(H(u))(t)| \leq M_{C} M_{1} \\
& \Longrightarrow\|\widetilde{C} H(x)\|_{\infty} \leq M_{C} M_{1}
\end{aligned}
$$

and, by the continuity of $C(t)$, as $t \rightarrow t_{k}$

$$
\begin{aligned}
& \left|(\widetilde{C} H(x))(t)-(\widetilde{C} H(x))\left(t_{k}\right)\right| \\
& \quad \leq M_{1}\left|C(t)-C\left(t_{k}\right)\right| \longrightarrow 0 .
\end{aligned}
$$

These, following Proposition 7, are enough to prove that $W x \in$ $B P C_{\Theta}[-r,+\infty)$ and $(W x)\left(t_{k}^{+}\right)-(W x)\left(t_{k}^{-}\right)=I_{k}\left(x\left(t_{k}\right)\right)$.

Moreover, it is not difficult to verify that $(\widetilde{C} H(x))^{\prime}(t)=$ $A S(t)(H(u)(t))$ for $t \geq 0$, and this is enought to prove that $(\widetilde{C} H(x))^{\prime}\left(t_{k}^{+}\right)=(\widetilde{C} H(x))^{\prime}\left(t_{k}^{-}\right)$so that one has

$$
(W x)^{\prime}\left(t_{k}^{+}\right)-(W x)^{\prime}\left(t_{k}^{-}\right)=J_{k}\left(x\left(t_{k}\right)\right) .
$$

From the hypotheses on $L$ one has

$$
L S(t) \eta=L w_{x}(t)=L(I x)(t)=L(J x)(t)=0,
$$

so, it follows from $\left(h_{5}\right)$ that

$$
\begin{aligned}
L(W x)(t)= & L(\widetilde{C} H(x))(t)+L S(t) \eta \\
& +L w_{x}(t)+L(I x)(t)+L(J x)(t) \\
= & L(\widetilde{C} H(x))(t)=(H(x))(t) .
\end{aligned}
$$

Moreover, $(\widetilde{C} H(x))^{\prime \prime}(t)=A(\widetilde{C} H(x))(t)$ and so, using Proposition 8, the fixed points of $W$ are solutions of $\left(\mathrm{P}_{\mathrm{F}}\right)$. It remains to prove that we can control the oscillations of $W x$ by a finite number of bounded functions.

We need to control only the oscillations of $\widetilde{C} H(x)$. If $0<$ $\tau_{1}<\tau_{2}$ (the other case are similar), we have

$$
\begin{aligned}
& \left|(\widetilde{C} H(x))\left(\tau_{1}\right)-(\widetilde{C} H(x))\left(\tau_{2}\right)\right| \\
& \quad \leq\left|C\left(\tau_{1}\right)-C\left(\tau_{2}\right)\right| M_{1} .
\end{aligned}
$$

So, by defining $\gamma(t):=M_{1} C(t)$ we have

$$
\begin{aligned}
& \left|(\widetilde{C} H(x))\left(\tau_{1}\right)-(\widetilde{C} H(x))\left(\tau_{2}\right)\right| \\
& \quad \leq \gamma\left(\tau_{1}\right)-\gamma\left(\tau_{2}\right) .
\end{aligned}
$$

Hence $W$ is a completely continuous operator. Following the same proof of Theorem 11 one can see that the set

$$
\zeta(W):=\left\{x \in \mathbb{R}^{n}: x=\lambda W x \text {, for some } 0<\lambda<1\right\}
$$

is bounded, so $W$ has fixed points that are solutions of $\left(\mathrm{P}_{\mathrm{F}}\right)$. This complete the proof.

Remark 13. The reader is referred to $[5,9,13,19]$ for some examples and applications.

\section{References}

[1] D. Baĭnov and P. Simeonov, Impulsive Differential Equations: Periodic Solutions and Applications, vol. 66 of Pitman Monographs and Surveys in Pure and Applied Mathematics, Longman Scientific \& Technical, New York, NY, USA, 1993.

[2] M. Benchohra, J. Henderson, and S. K. Ntouyas, Impulsive Differential Equations and Inclusions, vol. 2 of Contemporary Mathematics and Its Applications, Hindawi Publishing Corporation, New York, NY, USA, 2006.

[3] V. Lakshmikantham, D. D. Bainnov, and P. S. Simeonov, Theory of Impulsive Differential Equations, vol. 6 of Series in Modern Applied Mathematics, World Scientific, Teaneck, NJ, USA, 1989.

[4] A. M. Samoilenko and N. A. Perestyuk, Impulsive Differential Equations, vol. 14, World Scientific, River Edge, NJ, USA, 1995.

[5] X. Liu and A. R. Willms, "Impulsive controllability of linear dynamical systems with applications to maneuvers of spacecraft," Mathematical Problems in Engineering, vol. 2, pp. 277299, 1996.

[6] R. Sakthivel, E. R. Anandhi, and N. I. Mahmudov, "Approximate controllability of second-order systems with state-dependent delay," Numerical Functional Analysis and Optimization, vol. 29, no. 11-12, pp. 1347-1362, 2008.

[7] G. Arthi and K. Balachandran, "Controllability of damped second-order impulsive neutral functional differential systems with infinite delay," Journal of Optimization Theory and Applications, vol. 152, no. 3, pp. 799-813, 2012.

[8] D. N. Chalishajar, "Controllability of second order impulsive neutral functional differential inclusions with infinite delay," Journal of Optimization Theory and Applications, vol. 154, no. 2, pp. 672-684, 2012.

[9] E. Hernández, M. Rabello, and H. R. Henríquez, "Existence of solutions for impulsive partial neutral functional differential equations," Journal of Mathematical Analysis and Applications, vol. 331, no. 2, pp. 1135-1158, 2007.

[10] G. Infante and P. Pietramala, "Nonlocal impulsive boundary value problems with solutions that change sign, CP1124, Mathematical Models in Engineering, Biology, and Medicine," in Proceedings of the International Conference on Boundary Value Problems, A. Cabada, E. Liz, and J. J. Nieto, Eds., pp. 205-213, 2009.

[11] G. Infante, P. Pietramala, and M. Zima, "Positive solutions for a class of nonlocal impulsive BVPs via fixed point index," Topological Methods in Nonlinear Analysis, vol. 36, no. 2, pp. 263-284, 2010. 
[12] T. Jankowski, "Positive solutions of three-point boundary value problems for second order impulsive differential equations with advanced arguments," Applied Mathematics and Computation, vol. 197, no. 1, pp. 179-189, 2008.

[13] R. Sakthivel, N. I. Mahmudov, and J. H. Kim, "On controllability of second order nonlinear impulsive differential systems," Nonlinear Analysis. Theory, Methods \& Applications, vol. 71, no. 1-2, pp. 45-52, 2009.

[14] S. Sivasankaran, M. M. Arjunan, and V. Vijayakumar, "Existence of global solutions for second order impulsive abstract partial differential equations," Nonlinear Analysis. Theory, Methods \& Applications, vol. 74, no. 17, pp. 6747-6757, 2011.

[15] D. N. Chalishajar and F. S. Acharya, "Controllability of second order semi-linear neutral impulsive differential inclusions on unbounded domain with infinite delay in Banach spaces," Bulletin of the Korean Mathematical Society, vol. 48, no. 4, pp. 813-838, 2011.

[16] G. Marino, P. Pietramala, and L. Muglia, "Impulsive neutral semilinear equations on unbounded intervals," Nonlinear Functional Analysis and Applications, vol. 9, no. 4, pp. 527-543, 2004.

[17] G. Marino, P. Pietramala, and L. Muglia, "Impulsive neutral integrodifferential equations on unbounded intervals," Mediterranean Journal of Mathematics, vol. 1, no. 1, pp. 93-108, 2004.

[18] S. K. Ntouyas and D. O'Regan, "Existence results on semiinfinite intervals for nonlocal evolutions equations and inclusions via semigroup theory," Numerical Functional Analysis and Optimization, vol. 29, no. 3-4, pp. 419-444, 2008.

[19] J. P. Dauer and K. Balachandran, "Existence of solutions of nonlinear neutral integrodifferential equations in Banach spaces," Journal of Mathematical Analysis and Applications, vol. 251, no. 1, pp. 93-105, 2000.

[20] G. Marino, P. Pietramala, and H.-K. Xu, "Nonlinear neutral integrodifferential equations on unbounded intervals," International Mathematical Forum, vol. 1, no. 17-20, pp. 933-946, 2006.

[21] H. O. Fattorini, Second Order Linear Differential Equations in Banach Spaces, vol. 108 of North-Holland Mathematics Studies, North-Holland, Amsterdam, The Netherlands, 1985.

[22] J. A. Goldstein, Semigroups of Linear Operators and Applications, The Clarendon Press Oxford University Press, New York, NY, USA, 1985.

[23] C. C. Travis and G. F. Webb, "Cosine families and abstract nonlinear second order differential equations," Acta Mathematica Academiae Scientiarum Hungaricae, vol. 32, no. 1-2, pp. 75-96, 1978.

[24] H. Schaefer, "Über die Methode der a priori-Schranken," Mathematische Annalen, vol. 129, pp. 415-416, 1955.

[25] E. De Pascale, G. Lewicki, and G. Marino, "Some conditions for compactness in $B C(Q)$ and their application to boundary value problems," Analysis, vol. 22, no. 1, pp. 21-32, 2002. 


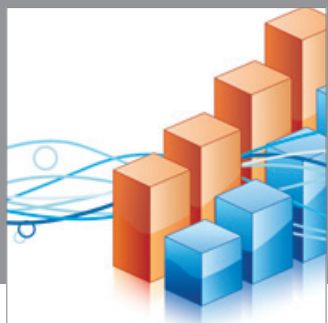

Advances in

Operations Research

mansans

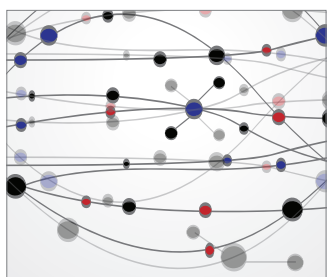

The Scientific World Journal
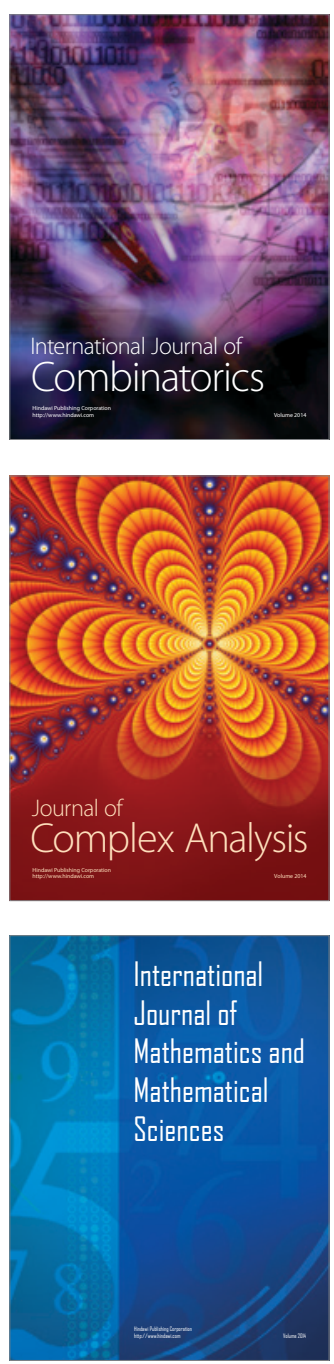
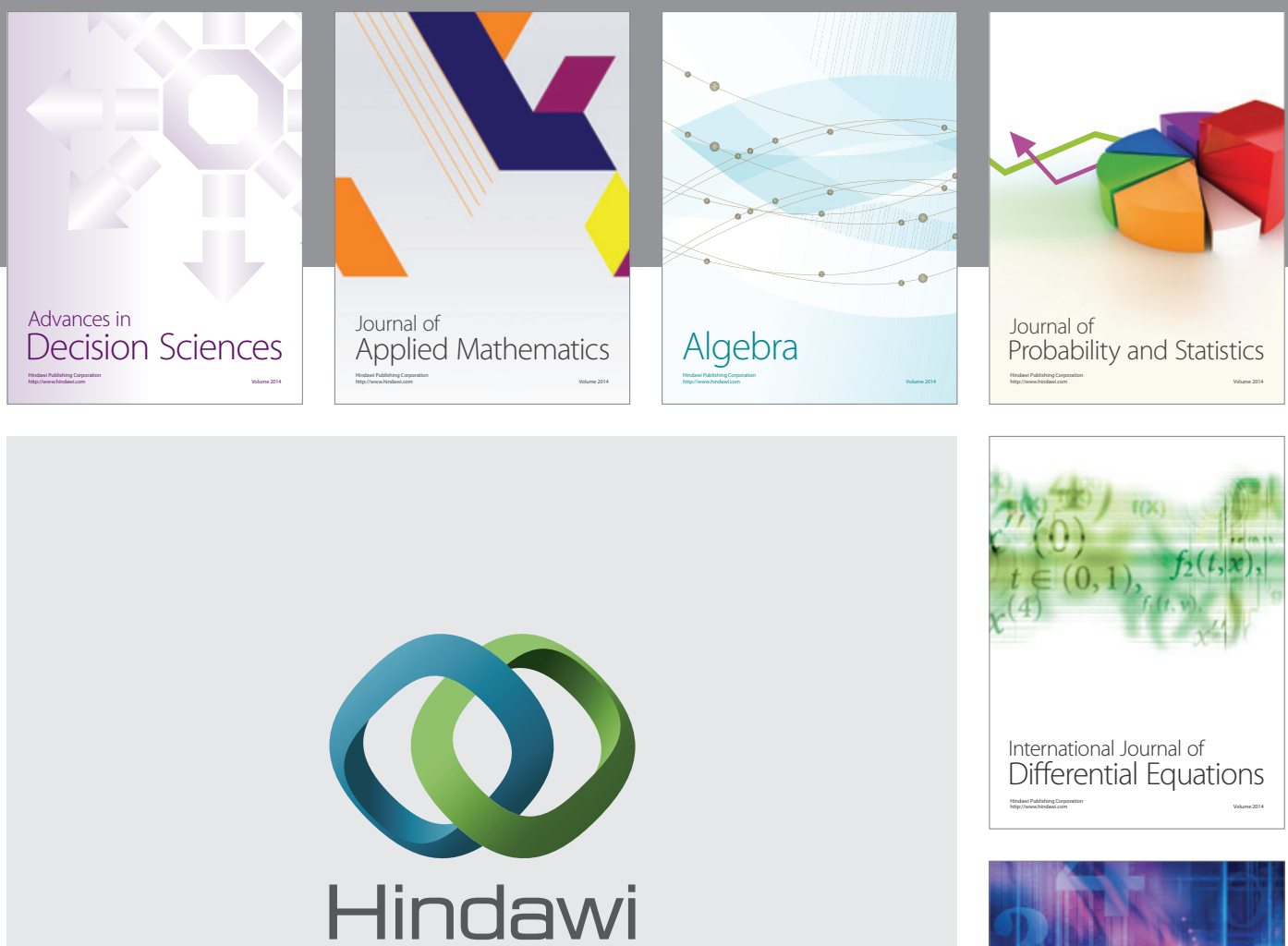

Submit your manuscripts at http://www.hindawi.com
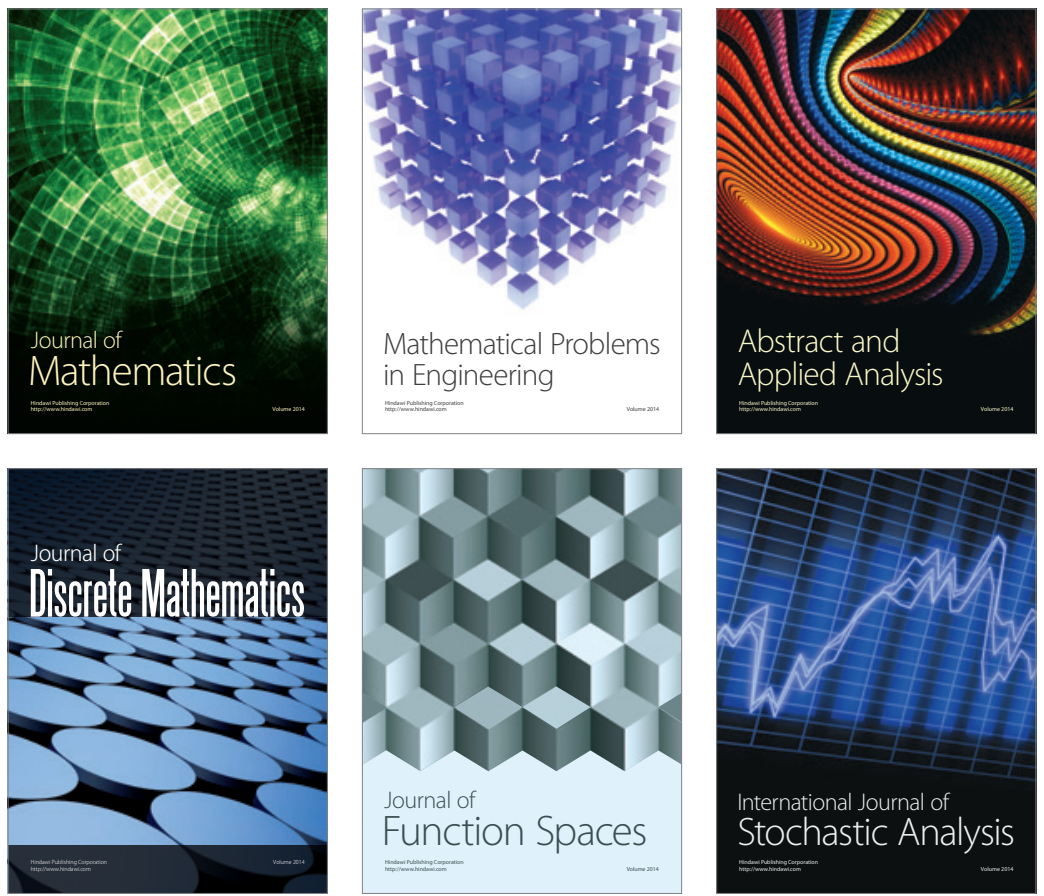

Journal of

Function Spaces

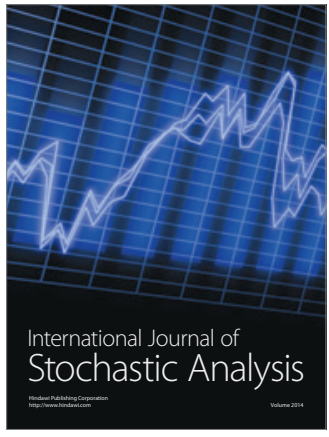

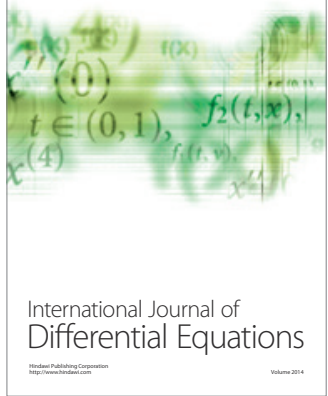
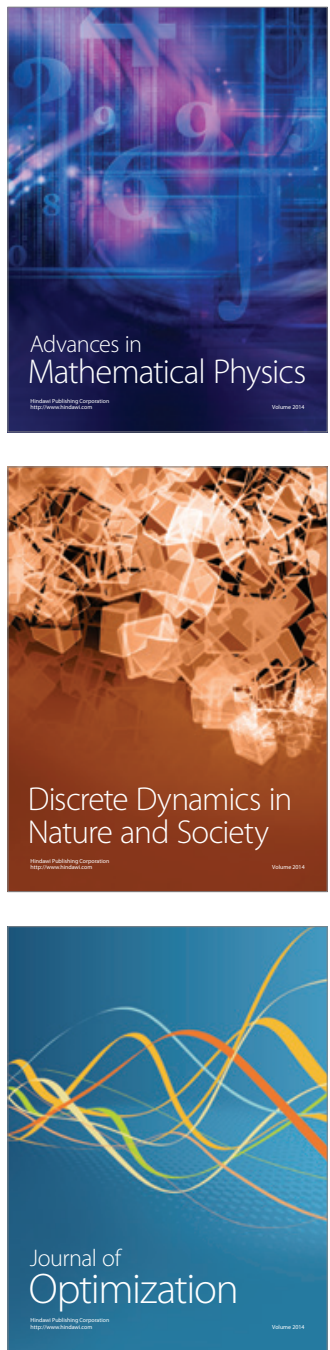